\title{
Phospholipase enzymes as potential biomarker for SARS CoV-2 virus
}

\author{
D.V.D. Hemalika \\ Department of Chemistry, Faculty of Natural Sciences, The Open University of Sri Lanka \\ DOI: 10.29322/IJSRP.11.01.2021.p10919 \\ http://dx.doi.org/10.29322/IJSRP.11.01.2021.p10919
}

\begin{abstract}
-
Severe acute respiratory syndrome corona virus 2 (SARS CoV-2) is the responsible pathogenic RNA virus which is responsible for current ongoing pandemic covid 19. This review provides an updated summary of the current knowledge of phospholipase enzymes and its role on SARS CoV-2 virus, discussing the reported evidence as a potential bio marker and future directions that could be used to develop PLAs as a therapeutic target for covid 19 pandemic.
\end{abstract}

Index terms- bio marker, covid 19, LpPLA2, SARS CoV-2, sPLA2, therapeutic target

\section{INTRODUCTION}

The covid 19 pandemic is a global health crisis which has grown exponentially to a disastrous scale. This is the greatest challenge we have faced since world war two.

Corona virus disease 2019 (covid 19) is an infectious disease which is caused by novel SARS CoV-2, Severe acute respiratory syndrome. Considering the epidemiology of covid 19, WHO declared it as a global pandemic on $11^{\text {th }}$ March 2020 [1]. Up to now more than 79,100,000 reported covid 19 infected cases with around 1700000 deaths have been reported [2].

SARS CoV-2 is the seventh corona virus that infect human. Until 2020, six corona viruses were known to infect human namely 229E, NL63 (genus Alpha-), OC43, HKU1, SARS-CoV, and MERS-CoV (Beta-)[3]. Among them in 2002 and 2012 we experienced severe out breaks of SARS-CoV and MERS with high mortality [4].Those two viruses and SARS CoV- 2 are beta corona viruses which infect lower respiratory tract and pneumonia in human. Corona viruses can cause common cold to more severe pneumonia condition in human. However compared to SARS CoV and MERS, covid 19 shows less severe pathogenesis, but very rapid transmission whole over the world [5].

This review summarizes the importance of phospholipase enzyme as therapeutic target for both noninfectious diseases and towards that its possibility to use as a potential biomarker for the covid 19 current pandemic situation.

\section{METHODOLGY}

Information for this review were obtained from previous research findings regarding phospholipase enzymes, SARS CoV-2 virus, use of phospholipase A2 (PLA2) as therapeutic targets for non-infectious diseases, role of PLA2 in corona viruses, studies of SARS CoV2 virus related to phospholipase enzyme from available literatures published in scientific databases such as Web of Science, Science Direct, PubMed, JSTOR and Google Scholar. Primary search terms like, PLA2, Covid 19, SARS CoV-2, enzymes as therapeutic targets are used to collect the information.

\section{STRUCTURE OF SARS COV-2}

Pathogenic RNA viruses become most important group of zoonotic disease transmission among all pathogens. Biological diversity, rapid inter transmission rates and epidemiology of recently developed Chikungunya(CHIKV) and Zika (ZIKV) viruses, Lassa fever, Ebolavirus, Middle East respiratory syndrome (MERS), SARS, and Influenza A virus (IAV) are all RNA viruses [5]. Coronaviruses 
belong to the family coronaviridae, (subfamily Coronavirinae), which represent major group of viruses which responsible for respiratory and neurological diseases [6].

Coronaviruses are single stranded positive sense RNA genome. Coronaviruses encode four major structural proteins, namely, spike $(\mathrm{S})$, membrane (M), envelope (E), and nucleocapsid (N) [5]. As its name implies this virus contain large, multifunctional transmembrane S glycoprotein on the virion surface, make crown like appearance [7]. Those glycoproteins are responsible for the viral attachment to the host cells. When we consider about our concern, SARS corona virus, in 2004 it is suggested that increasing international wildlife trade in countries like China and Vietnam may played an important role in SARS outbreak in 2002 [8]. Unfortunately, same incident is repeat in Wuhan, China in 2019 and now the outcome SARS CoV-2 is unable to anticipate by modern medical technologies even.

Using genome sequencing study of isolated viruses, it had been found that SAARS CoV- 2 is a new human infecting beta corona virus [9]. Covid 19 is a zoonotic disease and based on phylogenetic studies bats might be the original host of covid 19 virus. It was also found that Malayan pangolins (wild life mammal) are the intermediate host of SARS CoV-2 [10]. It had also been found that $\mathrm{s}$ glycoprotein which is contribute for the receptor binding is almost similar in both pangolin CoV and SARS CoV-2 [10].

\section{TREATMENT STRATEGIES FOR SARS CoV-2}

Covid 19 emerged in china and now it is rapidly spread all over the world. This public health burden is now become supreme challenge to whole universe. Therefore, strong investigations towards development of drugs and effective vaccines are highly needed for the society.

Up to date scientists in all over the world are eager to find therapeutic targets for this disastrous virus and few treatment strategies are identified and developed [11].

Severity and mortality of covid 19 patients are linked to excessive production of cytokines, called "cytokine storm" induce by the virus. It leads to wide spread tissue damage, multiple organ failure and death, Therefore it is important to target the inflammatory response as a therapeutic target for covid 19 virus [11].

In 2007, it had been shown another strategy that glycoproteins which is contain on the surface of the virus can be used as attractive target to develop as antiviral agents against corona viruses [12].

Developing neutralizing antibodies that targeting S protein on covid 19 virus surface, using oligonucleotides against covid 19 virus, Repurposing currently available antiviral medications [13] (viral polymerase and viral protease inhibitors [14]) against covid 19 viruses and development of vaccines (live vaccines with vector viruses, mRNA based vaccines, inactivated vaccines with viral proteins) are still under ongoing clinical trials [15].

\section{ENZYMES AS THERAPEUTIC TARGETS IN MEDICINE}

Enzymes are biological macromolecules, which are essential for all life. Enzyme catalysis is vital for the vast majority of biological reactions including synthesis of biomacromolecules (proteins, polysaccharides, and nucleic acids), intercellular communication and immune responses [16].

Although enzymes are crucial for most essential life processes, dysregulated enzyme activity can lead to disease conditions. As a result of these biological and pathophysiological implications, by modifying the action of enzymes, they become attractive targets for drug discovery. This strategy has been applied in the development of a substantial amount of antibiotic and antiviral drugs that exist today. In the year 2000, Hopkins and Groom reported that around $47 \%$ of drugs inhibit enzymes as their molecular targets. Nowadays major pharmaceutical companies are seeking new drugs through selective enzyme inhibition since enzymes are susceptible to be inhibited by small molecular weight drug-like molecules [17].

Viral enzymes also play a possible way to develop new anti-viral targets since each step of viral infection, viral enzymatic reactions are involved [13].

\section{ROLE OF LIPID METABOLISM IN VIRAL INFECTION}


Lipids have long been known as structural component in biological membranes of all living organisms. Lipids play a central role at different stages in viral replication including cell entry and exit by viruses. Virus entry is a specific process which is depend on viral surface proteins and host cell receptors[18]. Lipids also can function as host cell receptor for the viruses. Since lipids play a crucial role in viral life cycle it is good to investigate whether the enzymes which related to lipid metabolism can be used as drug target for viral infections.

Therefore, this lipid metabolic pathways become an important role in drug targets. Lipid related anti-viral strategies are promising since lipoids play a crucial role in viral replication. Identification of host directed anti-viral that inhibit host factors is most promising. It had been already reported that development of anti-viral drug for zika virus can drive through host directed anti-viral, since zika virus having a different aspects of lipid metabolism to complete their life cycle [19].

Since SARS CoV -2 virus replication is occur within the host cell, virus first should be enter to the host via intracellular membranes and create double membrane vesicles encoding with lipid bilayer and replicative organelles which are needed for the viral genome amplification [18].

Not only lipid mediators that produce through lipid metabolic pathways, but also the enzymes which involved is used as promising drug targets. Phospholipase enzymes may be considered as one such novel approach for fighting against SARS CoV-2 virus as well.

\section{PHOSPHOLIPASES ENZYME}

Phospholipases are a group of enzymes that lead to cleaving the various bonds in phospholipids, which are the major component of all biological membranes in living organisms.

Phospholipases are categorized based on the bond cleavage in phospholipid substrates.

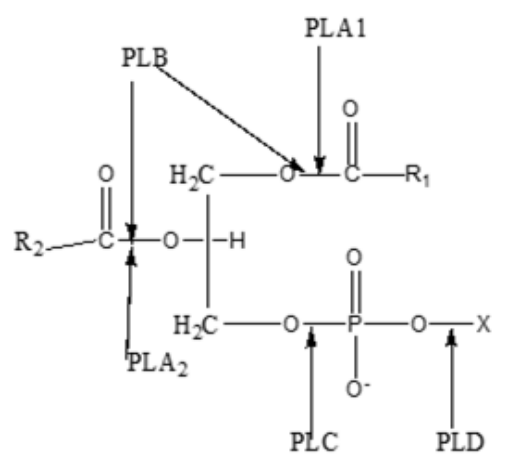

Figure 1: Site of action of various phospholipases on phospholipid. $\mathrm{X}=$ phospholipid common base (Choline, ethanol amine)

Based on the site of hydrolytic cleavage as depicted above, there are five types of phospholipases namely PLA1, PLA2, PLB, PLC, and PLD. Up to date, phospholipase A2 (PLA2) is the most extensively studied group of phospholipases [20].

PLA2 enzymes vary in size, function, location, substrate specificity, and calcium requirement. Based on the structure, catalytic mechanism, localization, and evolutionary relationships, PLA2s are subdivided into six subgroups as follows [21].

1. Cytosolic PLA2 - cPLA2

2. Secretory PLA2 - sPLA2 (Ca2+ dependent)

3. iPLA2 - Ca2+ independent PLA2

\section{LyPLA2 - lysosomal PLA2}

This publication is licensed under Creative Commons Attribution CC BY. 
5. PAFAH - PAF acetylhydrolase

\section{AdPLA2 - adipose PLA2}

Among them, sPLA2 is the first discovered group of PLA2 enzymes, which was discovered as a component of cobra venom [22]. PLA2 has been identified as one of the main components of animal venom. Elapidae and Viperidae family snakes having sPLA2 group IA, IIA or IIB as the main component in snake venom [23]. Snake venom PLA2s induce pathophysiological alterations in the victim by hydrolyzing phospholipids in membranes [23].

Among all existing isoforms of phospholipase enzymes, sPLA2 mainly play a major role in developing drug target as inhibitors since it involves in many inflammatory conditions [24].

Studies about this sPLA2 enzyme and its function, hold great importance since PLA2 catalyzes the release of arachidonic acid, which is believed to be the rate-limiting event in the generation of pro-inflammatory lipid mediators (prostaglandins, leukotrienes, lipoxins) and platelet-activating factor [25]. Release of these mediators initiates the pain, swelling, and other unpleasant symptoms we experience as part of an inflammatory response [26].

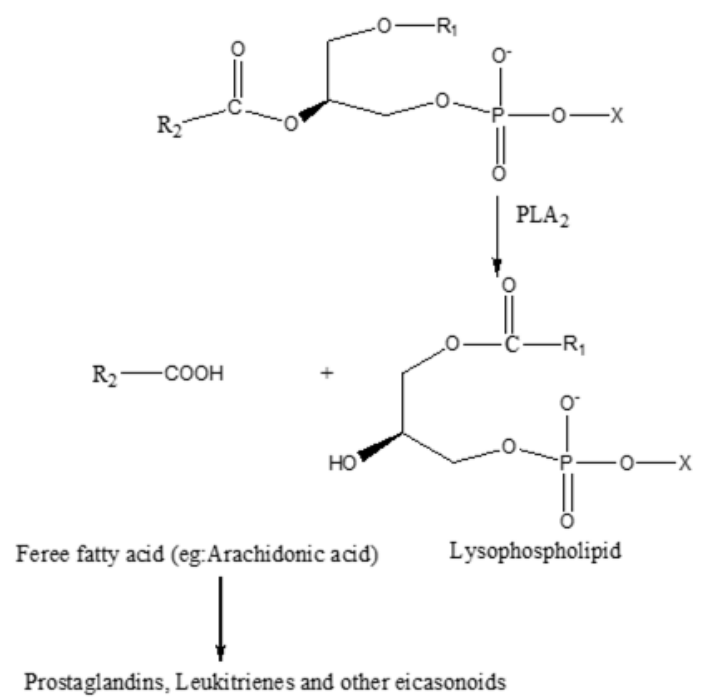

Figure 2: Phospholipid hydrolysis by sPLA2

Therefore, the inhibition of these phospholipase enzymes will be the good therapeutic target, as it leads to inhibit the production of inflammatory lipid mediators.

\subsection{SPLA2 ACTIVITY IN VARIOUS DISEASE STATES}

sPLA2 enzymes are responsible for a large variety of physiological functions as well in many disease conditions. They help to maintain membrane structure and function by removing oxidized and damaged phospholipids under physiological conditions [22].

It is important to discuss the cause of pathological conditions that are initiated by SPLA2. Lysophospholipids and free fatty acids (arachidonic acid) are the main products of hydrolysis of the phospholipid cell membrane. Arachidonic acid initiates the production of numerous metabolites through cyclooxygenase or lipoxygenase enzymatic pathways, especially eicosanoids including prostaglandins and leukotrienes, which mediate various pathological conditions [27].

Under pathological conditions, overexpression of sPLA2 activity can be observed in a variety of diseases. Especially increased activity of sPLA2 is associated with inflammatory diseases such as arthritis and sepsis [28], different types of cancer [29] and atherosclerosis [30]. 
These studies have implicated a role of sPLA2 IIA known as inflammatory sPLA2, in disease pathogenesis, since it promotes the production of inflammatory lipid mediators, mainly eicosanoids [31]. Moreover, it has been suggested that increased activity of sPLA2-IIA can be used as a biomarker for the detection of sepsis and the presence of bacterial infection in adults [27]. The increased sPLA2 activity has also been shown in the plasma or serum of patients with acute pancreatitis, septic shock, adult respiratory distress syndrome and inflammatory bowel diseases including Chron's disease and ulcerative colitis [22]. It had been shown that sPLA2 IIA, $\mathrm{V}$, and $\mathrm{X}$ was overexpressed in transgenic mice with atherosclerosis [30]. A recent study had shown that an increase in the activity of sPLA2 IIA was a significant risk factor in the occurrence of coronary artery disease.

sPLA2 IIA and sPLA2 $\mathrm{X}$ are the most studied sPLA2s in cancer so far [22]. It was determined that the sPLA2 $\mathrm{X}$ is expressed at high levels in colon cancer by promoting cell proliferation and releasing various lipid mediators in other steps in progression and development of cancer [33]. Several in-vitro studies had shown that human sPLA2 IIA activity is high in serum or in the tumor microenvironment of patients with prostate, esophageal and lung cancer cells [35]. The previous study had suggested that plasma sPLA2 IIA is a potential biomarker for lung cancer diagnosis since it is elevated in all most all lung cancer types [36]. sPLA2 IIA and sPLA2 $\mathrm{X}$ are the most studied sPLA2s in cancer so far. Several in-vitro studies had shown that human sPLA2 IIA activity is high in serum or in the tumor microenvironment of patients with prostate, esophageal and lung cancer cells [35]. It had been also reported that sPLA2 group II is overexpressed in a variety of human breast, gastric and hepatocytic carcinomas and prostate cancers [37].

Above studies were evidenced that sPLA2s are considered as pro-inflammatory enzymes and their inhibition has long been recognizing as a desirable therapeutic target.

\subsection{PHOSPHOLIPASE ACTIVITY IN VIRAL INFECTIONS}

Previous literature was cited that phospholipase enzyme act as key role of producing inflammatory lipid mediators in host during viral infections.

It had been reported that dengue virus (DENV) of neuroblastoma cell lines leads to increase in the activity of the sPLA2 enzyme leading to cell apoptosis. Increase in sPLA2 activity resulted in the increased production of prostaglandin E2 (PGE2), prostacyclin (PGI2), thromboxane and leukotriene, which could lead to the endothelial dysfunction leading to increased vascular permeability [9],[38].

It was demonstrated that dengue shock syndrome (DSS) patients had higher sPLA2 levels than healthy individuals [39]. Another study has shown that the sPLA2 activity was significantly higher in patients with DHF, especially in the very early phase of the illness. The activity of sPLA2 diminished towards 120-132 hours of illness and was similar to the sPLA2 activity seen in patients with DF [40].

Role of Phospholipace C (PLC) signaling had been reported for the bovine herpesvirus 1 infection which enhance the generation of inflammatory mediator reactive oxygen species [41].

It had been also evidenced that PLA2 host enzyme and lysophosphatidylcholins are contributed to form west Nile virus replication complex [42]. Moreover phospholipase D activity is stimulated by infection of influenza virus [43].

\subsection{ROLE OF PLA2 IN LUNG INFECTIONS AND RELATED RESPIRATORY PROBLEMS}

There are some evidence that elevated level of PLA2 is patients with lung infections and respiratory problems. Pulmonary surfactant is important to maintain alveolar stability by lowering surface tension along the alveolar epithelium. Destruction of this surface tension will results in lung injury (Acute Respiratory distress Syndrome ARDS) [44].

sPLA2 enzyme leads to hydrolyze phospholipid surfactant and destruction of surface tension. It had been found that inhibition of sPLA2 activity play a protective role in lung injury by maintaining surfactant integrity [45]. It was also reported that various sPLA2 isoforms are produced in lungs by macrophages and epithelial cells [46].

Using multicenter translational study including several pediatric and neonatal intensive care units suggested that sPLA2 might be the main cross road between inflammation and surfactant dysfunction in lungs [47].

Moreover it had been suggested that sPLA2 V and X participated to the lung injury by lipid mediator production and surfactant hydrolysis [48].There are patients with severe asthma showed increasing sPLA2 activity [49]. 


\section{STUDIES RELATED TO PHOSPHOLIPASE ENZYMES AND CORONAVIRUSES}

Studies related to PLA2 and covid 19 is already done by few scientists. As initial step it had been found that plasma sPLA2 level of moderate and acute covid 19 patients significantly increase compare to the covid 19 negative patients. They also observed the dramatic depletion of plasma phospholipids concentration with the elevation of sPLA2 [50]. That is may be due to hydrolysis of phospholipids and formation of lyso phospholipids. Resultant lysophospholipds may stimulate the cytokine production and this cytokine storm will lead the severity of SARS CoV-2.

This is further supported by recent finding that several circulating lipids and PLA2 activity can be used as potential biomarkers in the pathogenesis of covid 19. It is mainly due to the down regulation of glycerophospholipids and upregulation of lysophospholipids which produced by PLA2 activity [51]. It would suggest the strong influence of PLA2 in progression of pathogenesis of covid 19.

Previous reports were highlighted that cPLA2 $\alpha$ is critically involved in RNA replication of some viruses. This enzyme also important in corona virus replication by producing lysophospholipids that required to form double membrane vesicle formation, that is proven by both electron microscopy studies and lipidomic studies [52],[18].

In another study, age dependent increase of PLA2 Group IID in lungs of mice had been shown that it is linked with more than $80 \%$ lethality of SARS CoV in mice than mice with lacking PLA2 Group IID [53].

There are some studies done to evaluate the relationship between mortality of covid 19 patients with gender, sex, BMI, cholesterol level, Asthma and cardiovascular diseases. By analyzing the results of those studies, it was observed that PLA2 showed an important biological link in that patients.

One study had clearly shown that increased BMI levels were associated with higher risk of contracting SARS CoV-2 [54]. Higher BMI level is associated with increased sPLA2 activity in patients with stable asthma [49]. There is also an evidence that serum PLA2 level is higher in both asthma patients with and without attacks, when compared to the control group [56]. Therefore it can be suggested sPLA2 activity is interrelated in the patients having higher BMI and asthma towards the susceptibility of severe covid 19 [49].

While both men and women have the same prevalence to SARS CoV-2 without any gender discrimination, men is more susceptible to face more complications and death [55]. Study [49] was evidenced the inverse correlation of sPLA2 activity with vitamin c concentration in covid 19 patients. Interestingly the vitamin C concentration in plasma is lower in males than females [49]. It also links with the severity of covid 19 in males with the correlation of increasing sPLA2 activity and the decrease in vitamin C content.

LpPLA $_{2}$ is a member of the GVII family of PLA 2 enzymes. This enzyme was named for its ability to cleave the acetyl group from the $s n-2$ position of PAF, as well as its association with lipoproteins [57] . It was well established that increasing LPPLA2 is a reliable marker for the risk of cardiovascular events. It had been evidenced that LpPLA2 level upregulation is mainly found in nonhospitalized covid 19 patients. This abnormal increase LpPLA2 was observed in covid 19 re-positive patients as well [58], [59], [60]. Those patients are not showed promising symptoms of pneumonia, however sometimes they first experienced cardiovascular symptoms [61]. The limited medical care of these patients may follow up cardiovascular diseases.

Another study was revealed that Increasing rates of LpPLA2 were positively correlated with not only viral loads in patients with COVID-19 but also severity of pneumonia in non-COVID-19 patients. Therefore it could be suggested that increased levels of LpPLA2 in plasma could provide insights to higher mortality was seen in patients underlying comorbidities (e.g. hypertension, diabetes mellitus, cardiovascular disease) [62].

Moreover proteomics studies of covid 19 infected host cell showed a potential link with inflammatory response supported by increasing of PLA2 at 24h after virus infection [63].

Above studies revealed the contribution of phospholipase enzymes to SARS CoV-2 into some extent. However, it would be further investigated beyond the current understanding.

\section{CONCLUSION}

This review summarizes the PLA2 and its role in SARS CoV-2 infection. The increasing of phospholipase enzyme is linked with progression of disease from mild to severe and it is related with other associated complications like pneumonia, cardiovascular disease 
and towards the mortality. Therefore, this valuable information has unveiled potential antiviral targets that are now starting to be explored.

Further understanding of interfacial activation mechanisms of PLA2s on lipid surface will also uncover the new therapeutic target. Future studies with larger number of subjects is need to develop novel PLA2 inhibitors as therapeutic target for not only for covid 19, but also other related diseases. Optimization of existing PLA2 inhibitors will also be a good approach to develop therapeutics for not only infectious diseases but also for non-infectious disease.

\section{REFERENCES}

[1] WHO, "Listings of WHO's response to COVID-19," WHO, 2020. https://www.who.int/news/item/29-06-2020-covidtimeline.

[2] worldometer, “COVID-19 CORONAVIRUS PANDEMIC,” 2020. https://www.worldometers.info/coronavirus/.

[3] N. Zhu et al., "A Novel Coronavirus from Patients with Pneumonia in China, 2019," N. Engl. J. Med., vol. 382, no. 8, pp. 727-733, 2020, doi: 10.1056/nejmoa2001017.

[4] Y. Chen, Q. Liu, and D. Guo, "Emerging coronaviruses: Genome structure, replication, and pathogenesis," J. Med. Virol., vol. 92, no. 4, pp. 418-423, 2020, doi: $10.1002 / \mathrm{jmv} .25681$.

[5] M. A. Dhama K, Khan S, Tiwari R, Sircar S, Bhat S, Malik Y.S, Singh K P, Chaicumpa W, Aldana DKB, "Coronavirus Disease 2019-COVID-19," Clin. Microbiol. Rev., vol. 33, no. 4, pp. 1-48, 2020.

[6] A. Gaurav and M. Al-Nema, "Polymerases of coronaviruses: Structure, function, and inhibitors," in Viral Polymerases Structures, Functions and Roles as Antiviral Drug Targets, Elsevier Inc., 2019, pp. 271-300.

[7] David A.J. Tyrrell and Steven H. Myint., "Coronaviruses," in Medical microbiology, 1996, p. chapter 60.

[8] H. P. Bell D, Roberton S, "Animal origins of SARS coronavirus: Possible links with the international trade in small carnivores.," Philos Trans $R$ Soc L. B Biol Sci, vol. 359, no. 1447, pp. 1107-1114, 2004.

[9] R. Lu et al., "Genomic characterisation and epidemiology of 2019 novel coronavirus: implications for virus origins and receptor binding," Lancet, vol. 395, pp. 565-574, 2020, doi: 10.1016/S0140-6736(20)30251-8.

[10] K. Xiao et al., "Isolation of SARS-CoV-2-related coronavirus from Malayan pangolins," Nature, vol. 583, pp. 286-289, 2020, doi: 10.1038/s41586-0202313-x.

[11] R. L. Kruse, "Therapeutic strategies in an outbreak scenario to treat the novel coronavirus originating in Wuhan, China," F1000Research, vol. 9, no. 72, pp. 1-14, 2020, doi: 10.12688/f1000research.22211.2.

[12] F. G. U. M. van der Meer et al., "The carbohydrate-binding plant lectins and the non-peptidic antibiotic pradimicin A target the glycans of the coronavirus envelope glycoproteins," J. Antimicrob. Chemother., vol. 60, no. 4, pp. 741-749, 2007, doi: 10.1093/jac/dkm301.

[13] H. Ferriz and R. Buchet, "Coronavirus and Enzymes: What the Past Told us?," Open Enzym. Inhib. J., vol. 5, no. 1, pp. 1-1, 2020, doi: $10.2174 / 0218749402005010001$

[14] C.-H. Tsai, P.-Y. Lee, V. Stollar, and M.-L. Li, “Antiviral Therapy Targeting Viral Polymerase,” Curr. Pharm. Des., vol. 12, no. 11, pp. 1339-1355, 2006, doi: $10.2174 / 138161206776361156$.

[15] G. association of research based pharmaceutical Company, "Vaccines to protect against Covid-19, the new coronavirus infection," VFA, 2020. https://www.vfa.de/de/englische-inhalte/vaccines-to-protect-against-covid-19.

[16] C. GM., "The Central Role of Enzymes as Biological Catalysts," in The Cell: A Molecular Approach. 2nd edition, 2000.

[17] R. R, "Inhibitors of Secretory Phospholipase A2 Group IIA,” Curr. Med. Chem., vol. 12, no. 25, pp. 3011-3026, 2005.

[18] M. Abu-Farha, T. A. Thanaraj, M. G. Qaddoumi, A. Hashem, J. Abubaker, and F. Al-Mulla, "The role of lipid metabolism in COVID-19 virus infection and as a drug target," Int. J. Mol. Sci., vol. 21, no. 3544, pp. 1-11, 2020, doi: 10.3390/ijms21103544.

[19] M. A. Martín-Acebes, N. J. de Oya, and J. C. Saiz, "Lipid metabolism as a source of druggable targets for antiviral discovery against zika and other flaviviruses," Pharmaceuticals, vol. 12, no. 2, 2019, doi: 10.3390/ph12020097.

[20] M. . Aloulou, A, Ali Y.B, Bezzine, S, Gargouri, Y, Gelb, "Chapter 4 Phospholipases: An Overview," in Lipases and phospholipases: Methods and protocoal, methods in molecular biology, 2012, pp. 63-85.

[21] and B. S. C. Nhat D. Quach, Robert D. Arnold, "Secretory Phospholipase A2 Enzymes as Pharmacological Targets for Treatment of Disease," Biochem. Pharmacol., vol. 90, no. 4, pp. 338-348, 2015.

[22] V. M. Edward A. Dennis, Jian Cao, Yuan-Hao Hsu, "Phospholipase A2 Enzymes: Physical Structure, Biological Function, Disease Implication, Chemical Inhibition, and Therapeutic Intervention," Chem Rev, vol. 111, no. 10, pp. 6130-6185, 2012.

[23] Y. Zambelli, V.O., Picolo, G., Fernandes, C.A .H., Fontes, M.R .M., Cury, “Pain and Analgesia,” Toxins (Basel)., vol. 9, no. 406, pp. 1-27, 2017.

This publication is licensed under Creative Commons Attribution CC BY.

http://dx.doi.org/10.29322/IJSRP.11.01.2021.p10919

WWW.ijsrp.org 


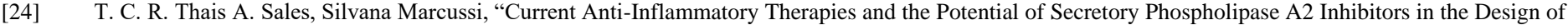
New Anti-Inflammatory Drugs: A Review of 2012 - 2018No Title," Curr. Med. Chem., vol. 27, no. 3, pp. 477-497, 2020.

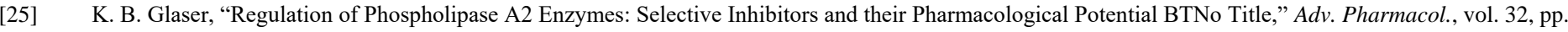
$31-66,1995$.

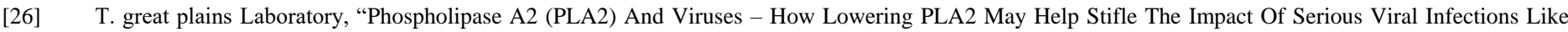
Coronavirus GENERAL,” 2020. [Online]. Available: https://www.greatplainslaboratory.com/pla2-coronavirus.

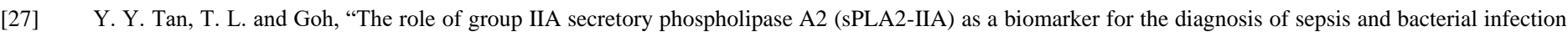
in 173 adults-A systematic reviewNo Title," PLoS One, vol. 12, no. 7, pp. 1-13, 2017.

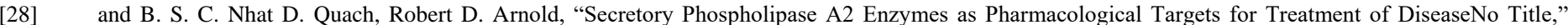
Biochem. Pharmacol., vol. 90, no. 4, pp. 338-348, 2015.

[29] V. Petan, T. and Brglez, "Secreted phospholipases A 2 in cancer: Diverse mechanisms of actionNo Title," Biochimie, vol. 107, pp. 114-123, 2014.

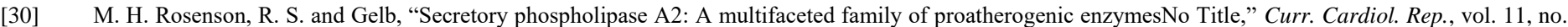
6, pp. 445-451, 2009.

[31] K. Murakami, M., Taketomi, Y., Sato, H., Yamamoto, “Secreted phospholipase A 2 revisitedNo Title,” J. Biochem., vol. 150, no. 3, pp. 233-255, 2011.

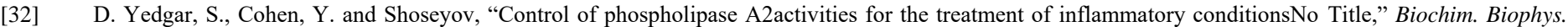
Acta - Mol. Cell Biol. Lipids, vol. 1761, no. 11, pp. 1373-1382, 2006.

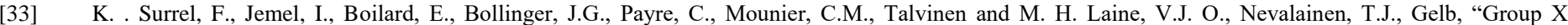
Phospholipase A 2 Stimulates the Proliferation of Colon Cancer Cells by Producing Various Lipid MediatorsNo Title," Mol. Pharmacol., vol. 76, no. 4, pp. 778-790, 2009.

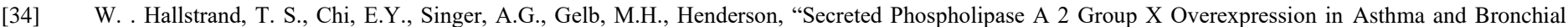
HyperresponsivenessNo Title,” Am. J. Respir. Crit. Care Med., vol. 176, pp. 1072-1078, 2007.

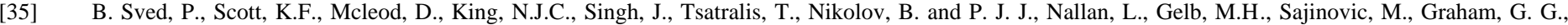
Russell, “Oncogenic Action of Secreted Phospholipase A 2 in Prostate CancerNo Title," Cancer Res., vol. 64, pp. 6934-6940, 2006.

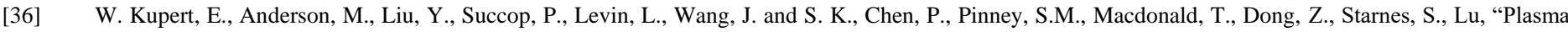
secretory phospholipase A2-IIA as a potential biomarker for lung cancer in patients with solitary pulmonary nodulesNo Title," Bio med Cent., vol. 11, no. 513, pp. 1-10, 2011.

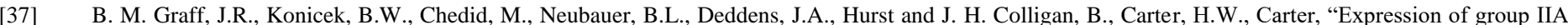
secretory phospholipase A2 increases with prostate tumor grade," Clin. Cancer Res., vol. 7, no. 12, pp. 3857-3861, 2001.

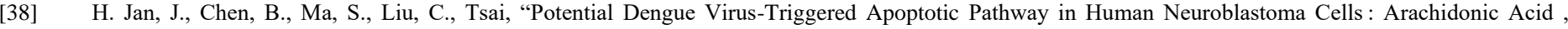
Superoxide Anion, and NF-KB are Sequentially Involved," J. Virol., vol. 74, no. 18, pp. 8680-8691, 2000.

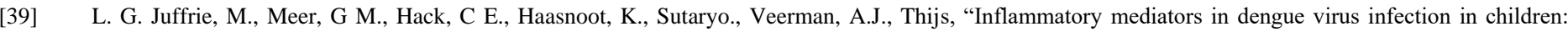
interleukin-6 and its relation to C-reactive protein and secretory phospholipase A2," Am. J. Trop. Med. Hyg., vol. 65, no. 1, pp. 70-75, 2001.

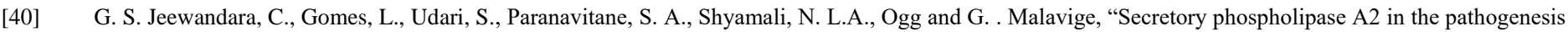
of acute dengue infection," Immunity, Inflamm. Dis., vol. 5, no. 1, pp. 7-15, 2017.

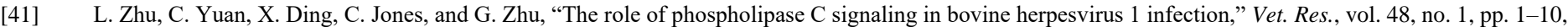
2017, doi: 10.1186/s13567-017-0450-5.

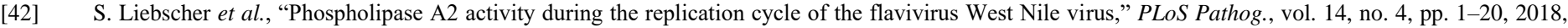
doi: 10.1371/JOURNAL.PPAT.1007029.

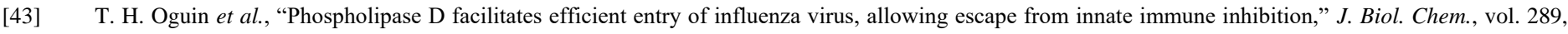
no. 37, pp. 25405-25417, 2014, doi: 10.1074/jbc.M114.558817.

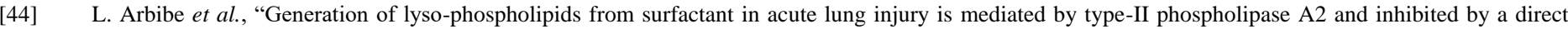
surfactant protein A-phospholipase A2 protein interaction,” J. Clin. Invest., vol. 102, no. 6, pp. 1152-1160, 1998, doi: 10.1172/JCI3236.

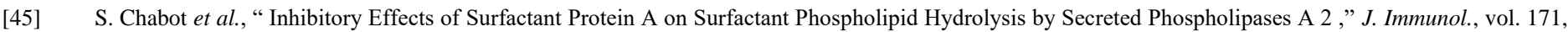
no. 2, pp. 995-1000, 2003, doi: 10.4049/jimmunol.171.2.995.

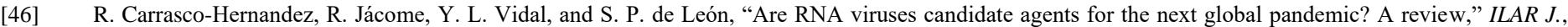
vol. 58, no. 3, pp. 343-358, 2017, doi: 10.1093/ilar/ilx026.

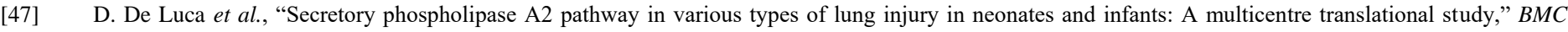
Pediatr., vol. 11, no. 1, p. 101, 2011, doi: 10.1186/1471-2431-11-101.

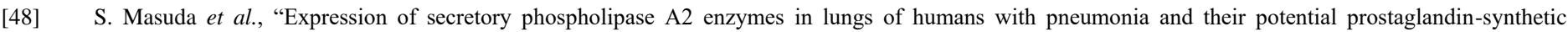
function in human lung-derived cells," Biochem. J., vol. 387, no. 1, pp. 27-38, 2005, doi: 10.1042/BJ20041307.

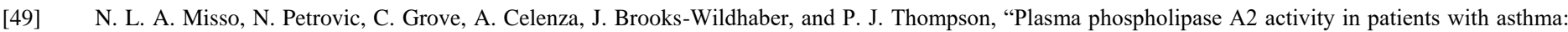
Association with body mass index and cholesterol concentration," Thorax, vol. 63, no. 1, pp. 21-26, 2008, doi: 10.1136/thx.2006.074112.

This publication is licensed under Creative Commons Attribution CC BY.

http://dx.doi.org/10.29322/IJSRP.11.01.2021.p10919 


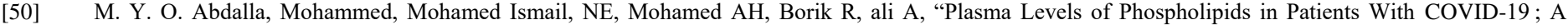
Promising Simple Biochemical Parameter to Evaluate the Disease Severity," 1811.

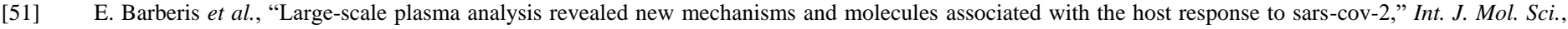
vol. 21, no. 22, pp. 1-25, 2020, doi: 10.3390/ijms 21228623 .

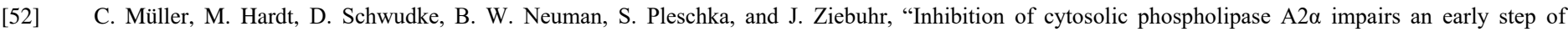
coronavirus replication in cell culture," J. Virol., vol. 92, no. 4, pp. 1-20, 2018, doi: 10.1128/jvi.01463-17.

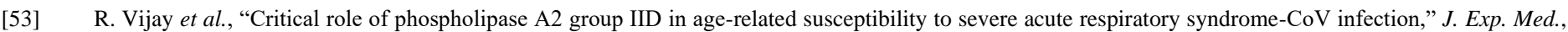
vol. 212, no. 11, pp. 1851-1868, 2015, doi: 10.1084/jem.20150632.

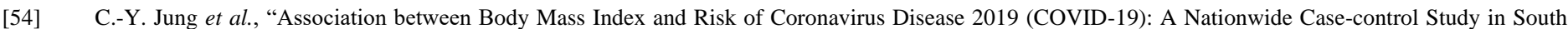
Korea," Clin. Infect. Dis., vol. xx, no. Xx Xxxx, pp. 1-8, 2020, doi: 10.1093/cid/ciaa1257.

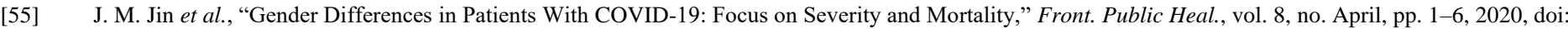
10.3389/fpubh.2020.00152.

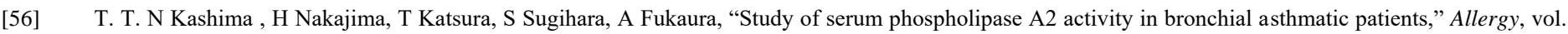
42, no. 6, pp. 723-727, 1993.

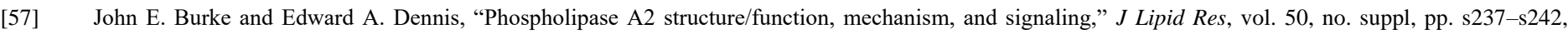
2009.

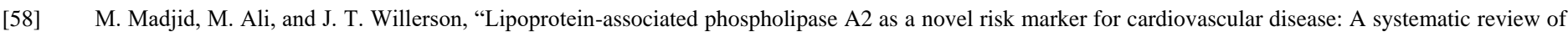
the literature," Texas Hear. Inst. J., vol. 37, no. 1, pp. 25-39, 2010.

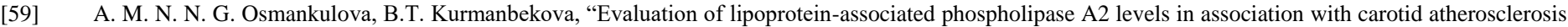
in patients with coronary artery disease," 2020.

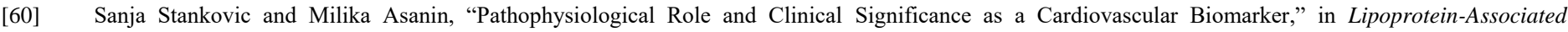
Phospholipase A2, no. intech open, 2016, pp. 113-135.

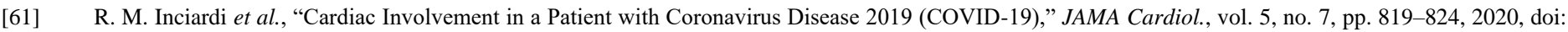
10.1001/jamacardio.2020.1096.

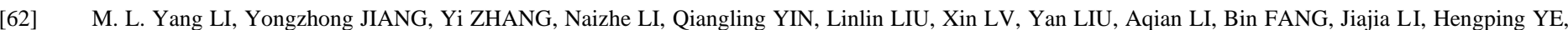
Gang YANG, Xiaoxian CUI, Yang LIU, Yuanyuan QU, Chuan LI, Jiandong LI, Dexin LI, Shiwen WANG, Zhongtao GAI, Faxian ZHAN, "Abnormal Upregulation of Cardiovascular Disease Biomarker PLA2G7 Induced by Proinflammatory Macrophages in COVID-19 patients," 2020.

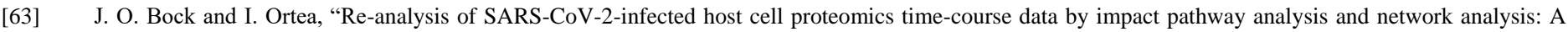
potential link with inflammatory response," Aging (Albany. NY)., vol. 12, no. 12, pp. 11277-11286, 2020, doi: 10.18632/aging.103524.

\section{AUTHORS}

First author - D.V.D. Hemalika, Ph.D, Faculty of Natural Sciences, The Open University of Sri Lanka dvhem@ou.ac.lk

Correspondence Author - D.V.D. Hemalika, Ph.D, Faculty of Natural Sciences, The Open University of Sri Lanka dvhem@ou.ac.lk, mobile no: +94711358415 